\title{
A new RICEs model with the global emission reduction schemes
}

\author{
WANG Zheng ${ }^{1,3^{*}}$, ZHANG Shuai $^{1,2}$ \& WU Jing ${ }^{1}$ \\ ${ }^{1}$ Institute of Policy and Management, Chinese Academy of Sciences, Beijing 100190, China; \\ ${ }^{2}$ Graduate University of Chinese Academy of Sciences, Beijing 100049, China; \\ ${ }^{3}$ East China Normal University, Shanghai 200062, China
}

Received February 17, 2012; accepted May 8, 2012; published online July 10, 2012

\begin{abstract}
Taking into account the fact of global economic integration, this paper improves the RICE model and the MRICES model, and establishes a new integrated assessment model MRICES-2012, which takes Ramsey utility as the standard of fairness. Based on the model, schemes which meet the global emission mitigation targets as well as the interests of developing countries are simulated to assess the international fairness of emission reduction. Therefore, a new feasible scheme is proposed, which can not only reach the Copenhagen Consensus but also ensure interests of every country. Specifically speaking, the US and Japan cut emissions $80 \%$ and $70 \%$ respectively by 2050 relative to the 1990 level; the EU and other developed countries cut $80 \%$ by 2050 relative to 1990 level; high human development countries cut $50 \%$ by 2050 relative to 1990 level; all above-mentioned countries start emission reduction from 2020 and keep emission on 2050 level by 2100; China begins emission reduction from 2030 and cuts emission $15 \%$ by 2050 and $25 \%$ by 2100 relative to 2005 level; medium human development countries keep emission on 2020 level by 2100 ; low human development countries do not take part in reduction on emission intensity and global emission.
\end{abstract}

MRICES model, reduction scheme, IAM, Copenhagen Consensus, climate protection

Citation: $\quad$ Wang Z, Zhang S, Wu J. A new RICEs model with the global emission reduction schemes. Chin Sci Bull, 2012, 57: 4373-4380, doi: 10.1007/s11434-012$5272-2$

The climate protection scheme is a key issue for global cooperation on climate protection. As the first phase of the commitment of the Kyoto Protocol is about to expire, many countries and organizations have raised the global emissions reduction schemes for the follow-up reduction program to combat global warming. World famous schemes include Stern scheme [1], Sørensen scheme [2], the UNDP scheme [3], and the UNEP scheme (http://www.unep.org/publications/ebooks/emissionsgapreport/appendices.asp). In China, there are also many studies about how China and other developing countries participate in global emission reduction $[4,5]$, among which the principle of equality on global per capita emissions or the cumulative carbon emissions are discussed as well $[6,7]$. The international situation, however, has changed a lot. Firstly, the world has experienced the economic crisis, and developing countries represented by BRICS bring the recovery of world economy. Suppression

*Corresponding author (email: wangzheng@ casipm.ac.cn) on BRICS's economy may lead to a long-term poverty worldwide, though the BRICS countries also face the responsibility of reducing emissions. Secondly, the Great Kanto Earthquake brings the nuclear crisis, which challenges the development of nuclear power. Thirdly, the international economic crisis of 2008 also shows that, in the case of global economic integration, the United States (US) and China are the locomotive of world economy, excessive inhibition on the economy of China and the US may bring about a worldwide recession. Therefore, a further emission reduction scheme needs to be studied. Since the RICE (the Regional Integrated model of the Climate and the Economy, regional-level climate-economic integrated assessment model) model [8] does not consider GDP spillovers and technology spillovers resulting from the global economic integration and endogenous technological change, the energy consumption reduction and emission reduction induced by economic development are neglected. So scheme assessments based on RICE are imperfect, which need to be 
improved.

Generally speaking, it is impossible to completely eliminate the influence from details or dynamic complexity, modeling can help us better to reduce these effects [9]. Popp [10] extends RICE, taking endogenous technological change into account, but the world is considered as a whole, without regard to international trade, difference of national economy growth and the rapid change in developing countries. As a result, the fairness of international emission reduction can not be evaluated. Wang et al. [11] developed the MRICES model with GDP spillovers and learning-bydoing technological change based on Wang's work. In this paper, we are going to improve the model based on MRICES.

A good climate protection scheme should measure the impact of climate change not only on developed countries but also on developing countries [12]. An optimized global emission reduction scheme is studied based on MRICES [13]. Unfortunately, the model divides the world into six regions as China, Japan, the US, the European Union (EU), the former Soviet Union and the rest of the world, in which developing countries are taken as a whole and technological system and development needs of developing countries are not considered. It is deficient. Based on the differences of national economies, this paper expands MRICES by dividing the world into eight countries and regions, as China, the US, the EU, Japan, high human development countries (HHDC), medium human development countries (MHDC), low human development countries (LHDC) and other developed countries (ODC), in accordance with the standard division of the 2010 Human Development Index in United Nations Human Development Reports (http://en.wikipedia. org/wiki/Human_Development_Index). The human development index is a comprehensive evaluation on life, education and income (country classification can be found in Table S1).

As we all know, most countries reached the agreement in Copenhagen Climate Conference in 2009 to hold the increase in global temperature below $2^{\circ} \mathrm{C}$. It has been recognized as the basis for international negotiation. On the other hand, the national commitments in Copenhagen are insufficient to achieve $2^{\circ} \mathrm{C}$ target by the end of the century [7]. China and other developing countries must participate in the global emission reduction. In this case, we need to study a fair and feasible scheme for global emission reduction.

\section{Model and data}

\subsection{Model improvement}

Following the RICE-MRICES architecture, the model is divided into macro-economic module, climate response module, human-environment coordination module and multiregional GDP spillover module. Compared with previous MRICES model, the improvements include new regional segments as eight countries and regions, multi-regional
GDP spillovers and learning-by-doing technological change, named MRICES-2012. In MRICES-2012, due to the refinement and improvement of regions, some parameters in MRICES-2012 should be re-estimated.

(i) GDP spillovers. In MRICES-2012, countries are linked with each other by GDP spillover and carbon emissions, based on each national macro-dynamic economic model. According to GDP spillovers theory [14,15], GDP spillovers between two countries (regions) can be described as

$$
\ln Y^{(i)}-\ln Y_{-1}^{(i)}=\sum_{j=1}^{7} v_{j}^{(i)}\left(\ln Y^{(j)}-\ln Y_{-1}^{(j)}\right)+g^{(i)},
$$

in which the superscript $i$ denotes the spillovers source country, $j$ represents other countries (regions) except the source country (region). $Y^{(j)}$ and $Y_{-1}^{(j)}$ denote national (regional) GDP in current and a period before. $v_{j}^{(i)}$ is the coefficient for spillover between country $i$ and $j, g^{(i)}$ is the growth rate of country (region) $i$.

In MRICES-2012 model, the national (regional) GDP of 1991-2009 in constant 2000 US\$ are from the World Databank (www.worldbank.org). The GDP of a country group is summed from national GDP. The model can be used to analyze the socioeconomic influence of climate policies with GDP spillovers scenarios.

(ii) Carbon emissions and temperature. In accordance with the RICE model, economic activities (measured in total output) and global warming (measured in surface temperature) are associated with each other in MRICES-2012. First, we have to connect emission with total output, so $E_{i, t}^{(c)}$ is

$$
E_{i, t}^{(c)}=\sigma_{i, t}\left(1-\mu_{i, c}\right) Y_{i, t}\left(\frac{A_{i, t}}{A_{i, t}^{*}}\right),
$$

in which $\sigma_{i, t}$ is the regional energy intensity; $\mu_{i, c}$ is the sum of emission reduction rate. $\sigma_{i, t}$, different from RICE, is regional specified and endogenous learning-by-doing technological change is introduced into MRICES [10], which can reflect regional emission more accurately.

MRICES-2012 gets the total output of various countries (regions) in the case of global economic cooperation with GDP spillovers, then figures out emission based on emission intensity and output. On the other hand, differentiated regional technological changes are considered in MRICES. In fact, there are technological spillovers among countries in economic globalization [16]. Due to technological spillovers working on developing countries, mainly by technological learning from developed countries, the speed of technological changes in developing countries is greater than that in developed countries, which mainly relies on technological innovation. Figure 1 portrays the emission intensity changes in China and the US. In theory, regardless of the differences of such technological changes, the emission in developing countries will be overestimated. A detailed model on technological 


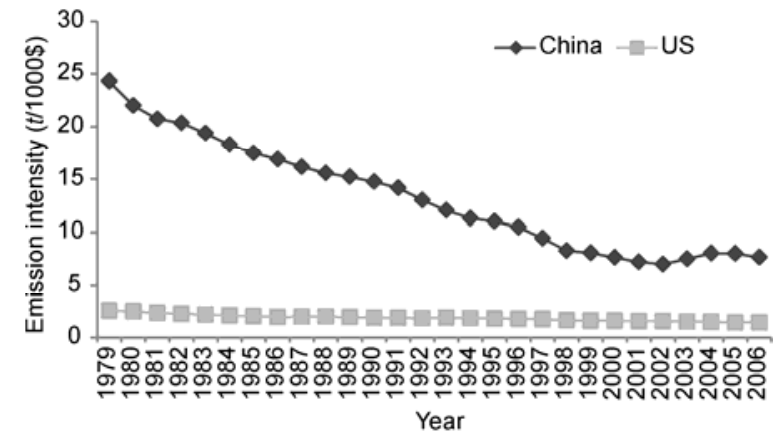

Figure 1 Emission intensity in China and the US (data source: CDIAC).

change can be found in Wang et al. [7].

Based on the national (regional) emission presented above, the world carbon emissions can be written as

$$
E_{t}=\sum_{i=1}^{8} E_{i, t}^{(c)}
$$

With the world carbon emissions, atmospheric carbon dioxide concentration can be figured out, which will increase the radiative forcing and finally lead to temperature rise, so that the economic activity and the global warming are linked. The composite details can be seen in Wang et al. [17].

The Keynes-Ramsey utility function is also adopted in RICE model with the implication that the utility is a function of consumption per capita. Because consumers are short-sighted in consumption, the utility of consumption changes with time preference $\rho$, which is defined as discounted rate. Taking into account the relative risk aversion $\tau$, the utility can be described as

$$
U_{i}(n)=\sum_{t=1}^{n}(1+\rho)^{-t} L_{i, t}^{(s)} \frac{\left(C_{i, t} / L_{i, t}^{(s)}\right)^{1-\tau}}{1-\tau},
$$

in which $C_{i, t}$ is the consumption at time $t$ subjecting to the resource constraints in production. The two parameters in eq. (4), $\rho$ and $\tau$, reflect the structure of the consumer's social welfare [18]. In recent years, a heated argument has appeared between Nordhaus [19] and Stern [20] on the value of $\rho$. We prefer to the opinion of Nordhaus [19], so 0.015 is taken for the value of $\rho$. Eq. (4) defines the accumulated Ramsay. Different from GDP, it not only takes into account the total amount of GDP, but also considers the per capita welfare, which makes it an indication of comprehensive national power. Only assessing the cost of emission reduction from GDP loss is unfair.

\subsection{Parameter estimation}

Based on the national GDP data in 1991-2009 from the World Databank, parameters in GDP spillovers model are estimated by regression, as shown in Table 1 . The $R^{2}$ of each regression is above 0.9 except that of China being about 0.78 , which proves the reliability of the estimation.

In Table 1, each number represents the spillover coefficient from the country (region) in the column to the country (region) in the row. In addition, it can be found in Table 1, the US, the EU, HHDC and MHDC promote the GDP growth in China with positive coefficients, while China also makes positive influences on the US, the EU, HHDC and MHDC, in which the promotion on HHDC is the biggest with MHDC following. It also shows that the US and China promote most countries' (regions') economies, reflecting the importance of keeping economic growth in China and the US in world development.

In the climate response module, we make out emission intensity in each country (region). According to national greenhouse gas emissions and GDP data, the rate of carbon emissions to output in base year 2004 is shown in Table 2.

Obviously, the carbon emissions intensity in Japan is the lowest, only $0.00006893 \mathrm{gC} / \$$. It is due to the advanced industrial structure in Japan and widely used nuclear energy. The earthquake in Japan in 2011 challenges the nuclear energy policy. This was followed by ODC, the EU and the US. Since China is the largest manufacturing country over the world, the carbon emissions intensity in China is relatively high. If carbon leakage is considered, the carbon emissions intensity in China will be only a little higher than that in MHDC.

\section{Convergence in accumulated carbon emissions per capita}

Many scholars have proposed that the fairness for emission reduction is to converge the accumulated carbon emissions

\begin{tabular}{|c|c|c|c|c|c|c|c|c|}
\hline & China & US & Japan & $E U$ & HHDC & MHDC & LHDC & ODC \\
\hline China & 0 & 0.329 & -0.647 & 0.262 & 0.553 & 1.738 & -1.03 & -0.925 \\
\hline US & 0.115 & 0 & 0.135 & 0.430 & 0.119 & -0.427 & -0.139 & 0.331 \\
\hline Japan & -0.116 & 0.069 & 0 & 0.540 & 0.441 & 0.861 & -0.571 & -0.245 \\
\hline EU & 0.070 & 0.326 & 0.799 & 0 & -0.341 & -0.802 & 0.608 & 0.443 \\
\hline HHDC & 0.266 & 0.163 & 1.181 & -0.616 & 0 & -1.105 & 1.1 & 0.507 \\
\hline MHDC & 0.231 & -0.162 & 0.636 & -0.400 & -0.305 & 0 & 0.55 & 0.517 \\
\hline LHDC & -0.289 & -0.111 & -0.896 & 0.644 & 0.644 & 1.169 & 0 & -0.607 \\
\hline ODC & -0.227 & 0.232 & -0.335 & 0.409 & 0.259 & 0.956 & -0.53 & 0 \\
\hline
\end{tabular}

Table 1 Parameter estimation for GDP spillovers 
Table 2 Carbon emissions intensity in each country (region) in 2004

\begin{tabular}{lc}
\hline & Carbon emissions intensity $(\mathrm{gC} / \$)$ \\
\hline China & 0.000718581 \\
US & 0.000147208 \\
Japan & 0.000068930 \\
EU & 0.000118826 \\
ODC & 0.000118820 \\
HHDC & 0.000476110 \\
MHDC & 0.000476790 \\
LHDC & 0.000190750 \\
\hline
\end{tabular}

per capita. The large number of historical emissions in developed countries makes them face a large number of emission reductions in the future. Therefore we propose scheme 0, in which the US, Japan, the EU and ODC cut emissions $80 \%$ by 2050 on 1990 level, the HHDC cut $50 \%$ by 2050 on 1990 level, all the above remains the total emission of 2050 by the year 2100 , while there is no emission reduction in developing countries. The accumulated carbon emissions per capita by 2100 in each country (region) are shown in Figure 2.

It can be found that, if developing countries do not participate in emission reduction, the cumulative per capita carbon emissions of developed countries are still significantly higher than those of the developing countries even if great reduction is taken in developed countries. Because the amount of historical emissions in the US and ODC is much larger than that in other countries. The accumulated emission per capita in the US is $269.25 \mathrm{tC}$, while that in China is only $176.1 \mathrm{tC}$, less than $35 \%$ of the US counterpart. Thus, even if developing countries do not participate in the emission reduction, the cumulative per capita carbon emissions in developing countries will also be significantly lower than those in the ODC.

The main drawback of scheme 0 is that it cannot achieve the "Copenhagen Consensus". In accordance with scheme 0 , the global atmospheric concentration of carbon dioxide equivalent is approximately $506 \mathrm{ppm}$ by 2050 and the temperature rise will be $2.35^{\circ} \mathrm{C}$ by 2100 . The accumulated Ramsay utility per capita is portrayed in Figure 3, which

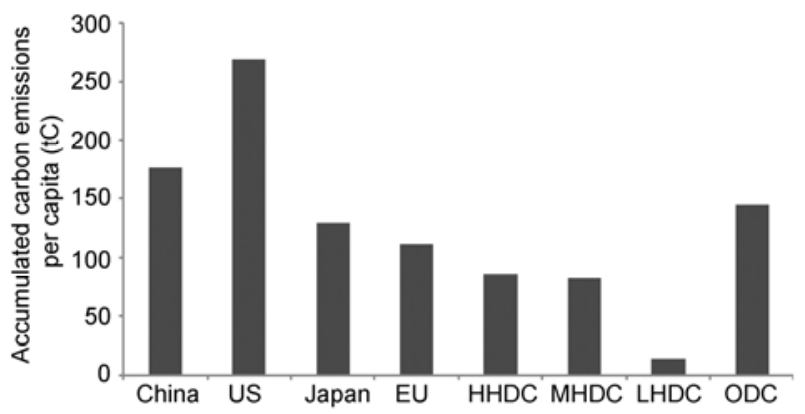

Figure 2 Accumulated carbon emissions per capita by 2100 in scheme 0. shows developing countries still have economic losses in scheme 0 . Therefore, the world has been unfair, it needs to examine how to control the exacerbation of the unfairness.

On the other hand, there are technological obstacles to further increase the emission reduction rates of developed countries to reduce their accumulated emission. In the world economic integration, excessive reduction in the US may also lead to the economic shrinking in China [17]. Thus, convergence in the cumulative carbon emissions per capita is unfeasible.

\section{Convergence in carbon emissions per capita}

Some scholars put forward the principle of equality in carbon emissions per capita, in which the carbon emissions per capita by 2100 in each country are convergent. Based on MRICES-2012, a number of possible emission reduction schemes are listed, and are searched one by one to look for the one meeting the Copenhagen Consensus and the principle of convergence in carbon emissions per capita. Consequently, we get the qualified scheme, in which all countries start to cut emissions in 2020, China cut emissions $18 \%$ and $15 \%$ on 2005 level by 2050 and 2100 respectively, the US, Japan, the EU and ODC cut emissions $82 \%, 65 \%, 67 \%$ and $71 \%$ on 1990 level by 2050 respectively, HHDC cut emissions $50 \%$ on 1990 level by 2050, MHDC keep equivalent with 2005 level, and low developing countries do not participate in emission reduction ${ }^{1)}$. For convenience, the scheme above is referred to as scheme 1 . The carbon emissions per capita by 2050 and 2100 in each country are shown in Figure 4, in which the emissions per capita in 2100 are higher than those in 2050 for the reason that emission increases when emission intensity keeps constant and output increases, leading to the increase of emissions per capita.

Based on Figure 4, the carbon emissions per capita in each country are almost equal to $0.78 \mathrm{tC}$ except $\mathrm{MHDC}$ and

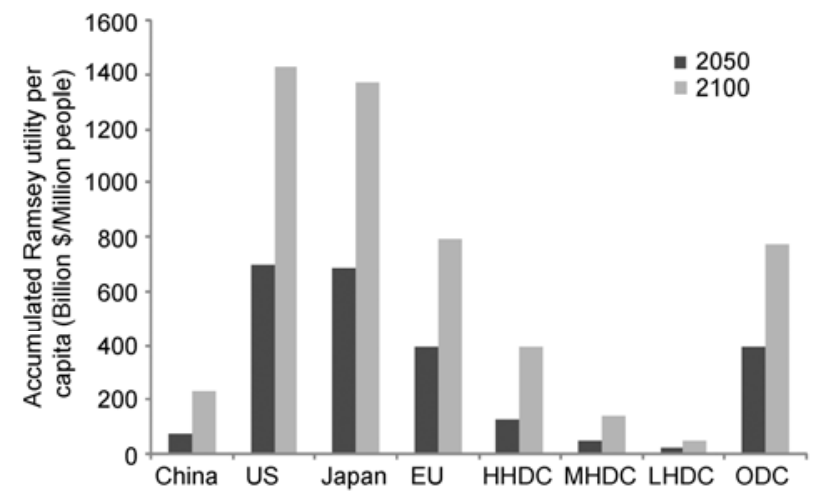

Figure 3 Accumulated Ramsey utility per capita in scheme 0.

1) Since the emissions in MHDC and LHDC are very low, and it is unlikely to increase rapidly in short-term, they are not listed in calibration of equality in carbon emission per capita. 


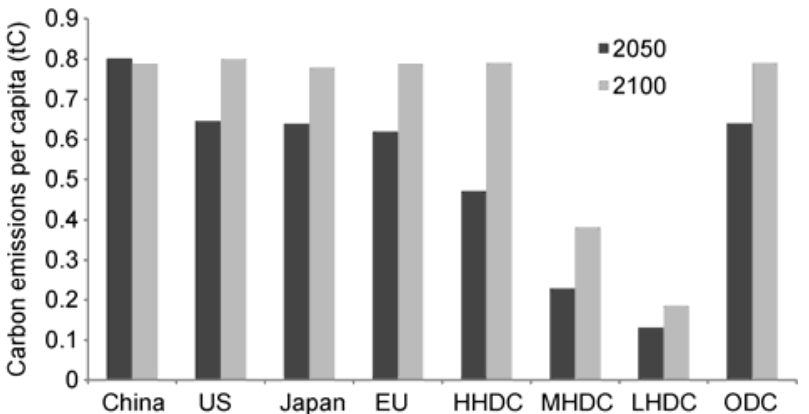

Figure 4 Carbon emissions per capita in scheme 1.

LDHC. Japan ranks the lowest with 0.78 tC per capita, while the US is the highest with $0.8 \mathrm{tC}$. The emissions per capita of China are $0.79 \mathrm{tC}$, almost equal to that of the other six countries. The variance of emissions per capita is 0.000043 , showing the convergence in different countries.

The global temperature rise by 2100 is $1.97^{\circ} \mathrm{C}$, and the $\mathrm{CO}_{2}$ concentration by 2050 is about $452 \mathrm{ppm}$, slightly higher than $450 \mathrm{ppm}$. So the convergence of carbon emissions per capita is basically a feasible solution. It must be pointed out that in this scenario, China and the developing countries actually make sacrifices when making the reduction target to keep emissions by 2050 on the level of 2005, which can be found from the accumulated emissions per capita in Figure 5. Additionally, due to the huge historical emissions in developed countries, the accumulated emission per capita in developed countries is still higher than that in developing countries even if they start reducing now. The highest emitter is the US with $268.7 \mathrm{tC}$, while the lowest is low developing countries with only $13.8 \mathrm{tC}$.

To further assess the influence of emission reduction, the change of accumulated Ramsey utility is evaluated. As shown in Figure 6, there is a big difference in accumulated Ramsey utility between countries (regions). Therefore, convergence in carbon emissions per capita is unfair from the view of accumulated Ramsey utility.

Of course, it is not appropriate to reach the equality on accumulated Ramsey utility. The target we want to achieve is to increase the accumulated Ramsey utility of developing countries while not to decrease the utility of developed countries when comparing emission reduction schemes with $\mathrm{BAU}$, for reaching the balanced development over the

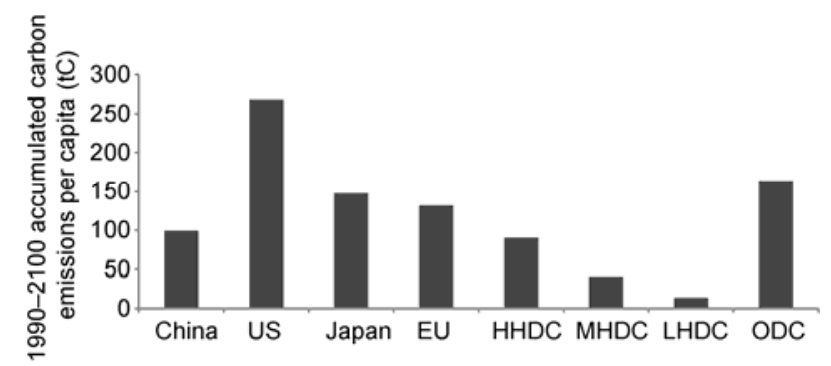

Figure 5 Accumulated carbon emissions per capita by 2100 in scheme 1.

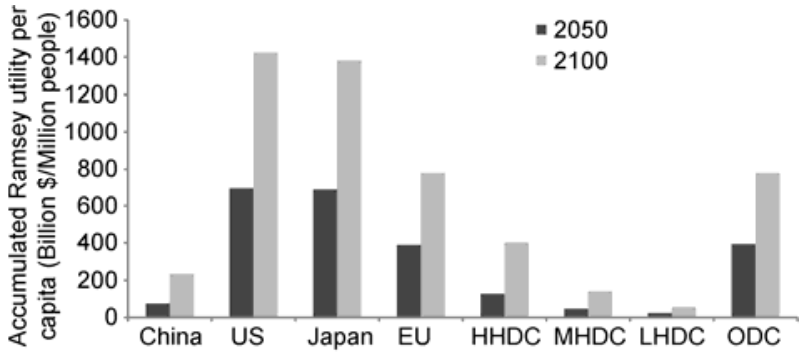

Figure 6 Accumulated Ramsey utility per capita in scheme 1.

world. Figure 7 shows the change of accumulated Ramsey utility in scheme 1 related to BAU, which indicates that the accumulated utility increases by 2100 though it losses by 2050, except MHDC and LHDC. The change of accumulated utility of the US is minus, implicating that no emission reduction is beneficial for the US's development, but the accumulated Ramsey utility and utility per capita of the US are still existent in the first place (Figure 6). So emission control should be carried out in the US for the common interests of the world.

\section{The scheme with the global economic growth}

The principle of convergence in carbon emissions per capita has its own rationality, while the global economic growth should be regarded when taking emission reduction. Taking into account that the US and China are big manufacturing countries and the world's economic leaders, the suppression of the carbon emissions of China and the US may bring global economic disaster, and should therefore allow slightly higher carbon emissions per capita in China and the US. On the other hand, the earthquake in Japan shows that large-scale nuclear power is not suitable for Japan considering Japan's geographical situation. The reduction strength should be relaxed in Japan.

Therefore, with several experiments a new scheme is obtained based on MRICES-2012, named scheme 2. Every country takes two-stage emission reduction in scheme 2 . China starts emission reduction from 2030 and reduces emissions $15 \%$ by 2050 on 2005 level, and reduces $25 \%$ by 2100 on 2005 level. All developed countries and HHDC start emission reduction from 2020 . The US reduces $80 \%$ by

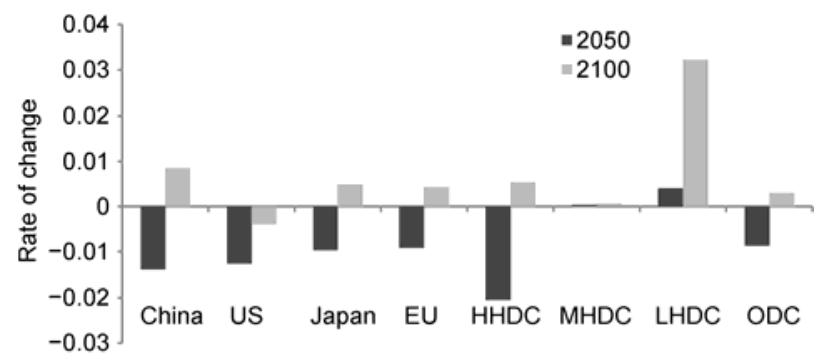

Figure 7 The change of accumulated Ramsey utility in scheme 1. 
2050 on 1990 level and keeps the same by 2100 . The EU and ODC reduce $80 \%$ by 2050 on 1990 level and keep the same by 2100 . Japan reduces $70 \%$ by 2050 on 1990 level and keeps the same by 2100 . MHDC peak $1.387 \mathrm{GtC}$ by 2020 via emission intensity reduction and keep the same by 2100. LHDC do not participate in any reduction. Since the nuclear power is not suitable for Japan, the emission reduction of Japan is relatively lower than that of other countries.

In scheme 2, the global carbon dioxide concentration by 2050 is $409.5 \mathrm{ppm}$, nearly equal to $449 \mathrm{ppm} \mathrm{CO}_{2}$ equivalent. The global temperature rise is $1.95^{\circ} \mathrm{C}$ by 2100 . The carbon emissions per capita for each country (region) are shown in Figure 8, in which the emissions per capita of the US and China are $0.685 \mathrm{tC}$ and $0.696 \mathrm{tC}$ respectively, ensuring the economic development in the two countries. The emissions per capita in Japan, the EU, ODC and HHDC are 0.501, $0.359,0.407$ and $0.451 \mathrm{tC}$, respectively. The advantage of scheme 1 is preserved, which can lead to a better economic growth. If China starts emission reduction from 2020 when keeping emission reduction targets of other countries, the global temperature rise will be $1.94^{\circ} \mathrm{C}$, showing a negligible influence on global mitigation targets from the reduction timing of China. If China starts emission reduction from 2030 , the 20-year average emission reduction rate is about $1.41 \%$, while the 30 -year average emission reduction rate is $0.4 \%$ if it starts reduction from 2020 , so the later the better is for economic development.

The Copenhagen Consensus is achieved in scheme 2 . Different from scheme 1, the emissions per capita by 2050 and 2100 vary among countries (regions), in which the levels in the US and China are about $0.7 \mathrm{tC}$ supporting their development. The emissions of MHDC and LHDC are not constrained relative to 2005 level, but their levels of emissions per capita are still low. It puts down to the large population base and the underdevelopment. So it is reasonable not to take part in emission reduction for the two groups.

In addition, the national (regional) accumulated carbon emissions also change in scheme 2, as shown in Figure 9.

In scheme 2, there are still differences in the accumulated carbon emissions per capita in developed and developing countries. Relative to scheme 1, the differences of the accumulated carbon emissions per capita between China and Japan, the EU and ODC decrease obviously. In scheme 1, the differences between China and the three countries

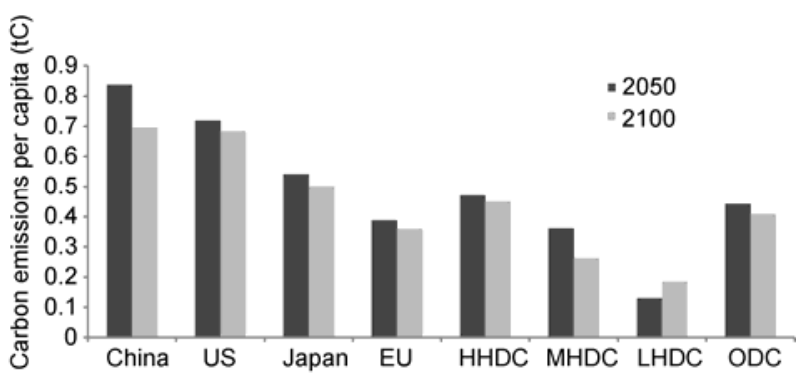

Figure 8 Carbon emissions per capita in scheme 2.

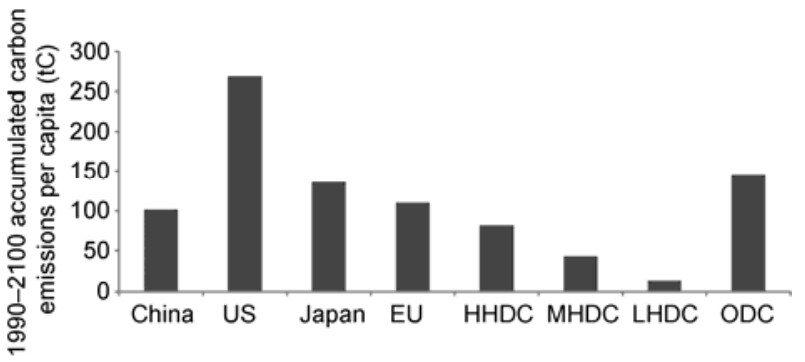

Figure 9 Change of accumulated carbon emissions per capita by 2100 in scheme 2.

(regions) are 48.23, 32.60 and $64.20 \mathrm{tC}$ respectively, which fall to $34.80,8.81$ and $43.76 \mathrm{tC}$ respectively. In other words, scheme 2 alleviates the large gap of accumulated carbon emissions per capita between China and developed countries in scheme 1 , so it is a compromise between the principle of convergence in carbon emissions per capita and accumulated carbon emissions by 2100 .

It should be noted that the accumulated Ramsey utility per capita and the rate of Ramsey utility change in scheme 2 are shown in Figures 10 and 11, respectively. According to Figure 10, there is no remarkable difference between scheme 2 and scheme 1 . The Ramsey utilities of Japan, the EU and ODC decrease slightly.

When focusing on the rate of Ramsey change, as shown in Figure 11, the change rate of the US is negative, but its carbon emissions per capita and the accumulated carbon emissions per capita still take the first place over the world (Figure 9). In scheme 2, the accumulated Ramsey utility of developing countries increases and that of developed countries does not decrease, which meets the target of emission reduction to reach a balanced development over the world.

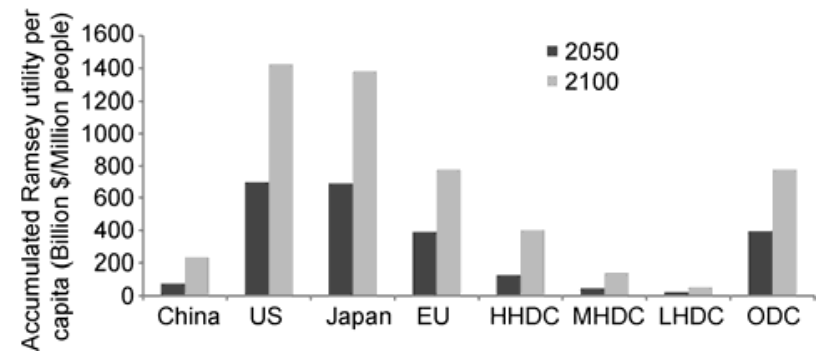

Figure 10 The Ramsey utility per capita in scheme 2 .

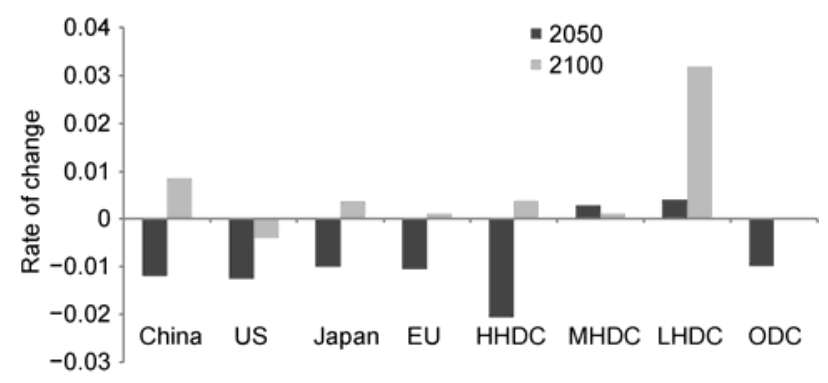

Figure 11 Rate of change of Ramsey utility in scheme 2. 
Obviously, schemes 1 and 2 are feasible for reduction. But the carbon emissions per capita and accumulated carbon emissions per capita are compromised in scheme 2 compared with scheme 1 , and scheme 2 meets the target of emission reduction much better from the perspective of Ramsey utility and the rate of change of Ramsey utility.

\section{Comparison of carbon emissions in different schemes}

There is a difference between schemes 1 and 2 in the global carbon emissions. As shown in Figure 11, the global carbon emissions begin to decrease from 2020 due to emission reduction actions taken in each country (region). However, since the diverse timing and strength for emission reduction, the trend of global carbon emissions growth differs after 2050. If the principle of convergence in carbon emissions per capita by 2100 is taken, a relatively fast increase is observed in the global carbon emissions growth. By contrast, if scheme 2 is taken, the global carbon emissions are almost stable after 2050, in which the slight increase is attributed to the emission from LHDC when they do not participate in emission reduction. The global carbon emissions by 2100 are 5.96 and $4.26 \mathrm{GtC}$ in schemes 1 and 2 respectively.

There is a distinct advantage in scheme 2 considering the global carbon emissions relative to that of scheme 1 . The 2011-2100 accumulated carbon emissions drop to 448.8 $\mathrm{GtC}$ from $471.1 \mathrm{GtC}$. Therefore, scheme 2 is much reasonable based on this criterion.

The global GDP is also different in the two schemes. Compared with the global GDP of 2006, the global GDP by 2100 in scheme 1 increases 11.88 times, 11.885 times in scheme 2, a little bit higher than that in scheme 1 . The annual GDP in the two schemes is shown in Figure 13. It can be found that GDP in the two schemes is almost overlapped, meaning the same growth trend of GDP in the future. Similarly, the accumulated GDP and Ramsey utility in scheme 2 are also higher than that in scheme 1 . Scheme 2 gets more emission reduction and less temperature rise, and the economic efficiency is better than that of scheme 1 . So with regard to loosening the emission reduction burdens on the

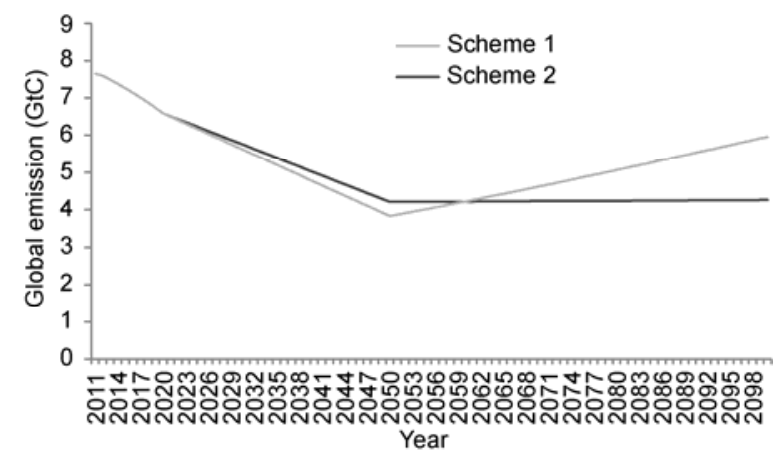

Figure 12 The global carbon emissions in schemes 1 and 2.

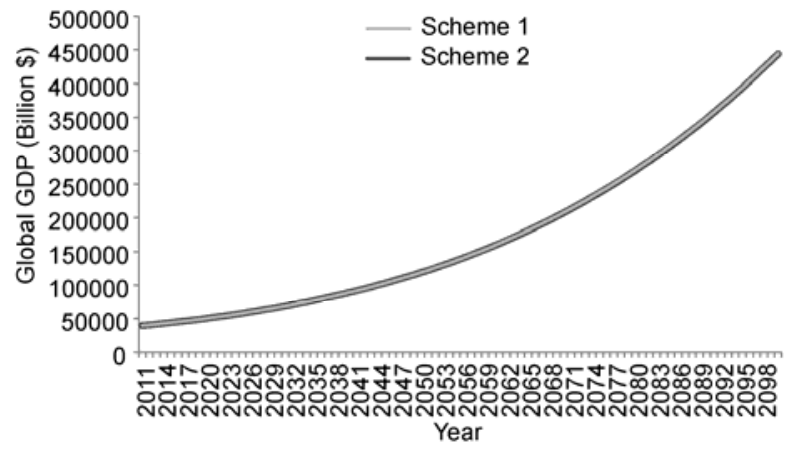

Figure 13 GDP comparison between two schemes (the economic growths are almost overlapped)

leading countries, scheme 2 is in line with global interests, in which the economic growth will not be damaged by temperature rise and no economic risk results from excessive reduction in some countries. It can be referred as the scheme with efficient economic growth.

In addition, in scheme 1 the carbon emissions of China are $1.255 \mathrm{GtC}$ by 2050 and $1.301 \mathrm{GtC}$ by 2100 , which are $18 \%$ and $15 \%$ lower than 2005 level respectively. The carbon emissions of the US in scheme 1 are $0.235 \mathrm{GtC}$ by 2050 and $0.31 \mathrm{GtC}$ by 2100 , which are $82 \%$ and $77 \%$ lower than 1990 level respectively. In scheme 2 , the emissions of China are $1.3 \mathrm{GtC}$ by 2050 , which is $15 \%$ lower than 2005 level. The emissions of the US are $0.262 \mathrm{GtC}$ by 2050 , which is $80 \%$ lower than that of 1990 level. The emissions of MHDC countries are $1.387 \mathrm{GtC}$ by 2100 , which is 1.58 times that 2005 level. But focusing on accumulated carbon emissions per capita, it are 101.648 tC in China which are much lower than $268.5 \mathrm{tC}$ in the US.

Therefore, the Copenhagen Consensus, in which the $\mathrm{CO}_{2}$ concentration by 2050 is below $450 \mathrm{ppm}$ and the temperature rise by 2100 is below $2^{\circ} \mathrm{C}$, can be reached in both schemes. Scheme 2 can boost the global economic growth much more effectively. In fact, MRICES-2012 can find many other schemes, but scheme 2 is one of the appropriate schemes for global climate protection.

\section{Conclusion}

This paper establishes an integrated assessment model for climate change based on RICE model, and introduces the global GDP spillovers and technological change into the model.

In the newly-developed MRICES-2012 model, the world is divided into 8 countries (regions) and is suitable for climate policy simulation. The typical RICE model does not take the global economic integration into account, so it overestimates the carbon emissions of China and MHDC and underestimates the influences of emission reduction in China and the US.

Based on MRICES-2012, global emission reduction 
schemes are discussed in accordance with Copenhagen Consensus, in which the global temperature rise by 2100 should be below $2^{\circ} \mathrm{C}$.

With the simulation on convergence in accumulated carbon emissions per capita, convergence in carbon emissions per capita and the scheme with global economic growth are assessed on MRICES-2012, the results show that it is impossible to control temperature rise below $2^{\circ} \mathrm{C}$ if only developed countries are engaged in emission reduction. The scheme of convergence in carbon emissions per capita, which meets the Copenhagen Consensus, can be retrieved on MRICES-2012, but the scheme is not fair for developing countries. Therefore, a new scheme with global economic growth is presented in this paper, which can not only meet Copenhagen Consensus but also can reach a better economic efficiency and fairness for developing countries.

The scheme with global economic growth can be specified as follows: China starts emission reduction from 2030 and cuts emissions $15 \%$ and $25 \%$ by 2050 and 2100 respectively on 2005 level; all developed countries and HHDC start emission reduction from 2020; the US cuts emissions $80 \%$ by 2050 on 1990 level and keeps emission on 2050 level by 2100 . The EU and ODC cut $80 \%$ by 2050 on 1990 level, and keep the same by 2100. Japan cuts emissions 70\% by 2050 on 1990 level, and keeps emission on 2050 level by 2100. HHDC peak in 2020 with $1.387 \mathrm{GtC}$ emissions and keep on the level by 2100. LHDC do not take part in reduction on emission intensity and global emission. Under this scheme, the global $\mathrm{CO}_{2}$ concentration by 2050 is 409.516 $\mathrm{ppm}$, equal to $449 \mathrm{ppm}$ equivalent, and the temperature rise by 2100 is $1.94^{\circ} \mathrm{C}$, reaching the $2^{\circ} \mathrm{C}$ target by 2100 .

This work was supported by the National Basic Research Program of China (2012CB955804) and the Strategic Priority Research Program of the Chinese Academy of Sciences (XDA05150500).

1 Stem N. Stem review on the economics of climate change. Report to the Prime Minister and the Chancellor of the Exchequer on the Economics of Climate Change, 2006
2 Sørensen B. Pathways to climate stabilisation. Energ Policy, 2008, 36: 3505-3509

3 UNDP. Fighting Climate Change: Human Solidarity in a Divided World. Human Development Report 2007/2008

4 Ding Z L, Fu B J, Han G X, et al. Brief Introduction to a cluster of projects of "research in key issues of international negotiation with regard to coping with climate change" by CAS (in Chinese). Bull Chin Acad Sci, 2009, 24: 8-17

5 Jiang K J, Hu X L, Liu Q, et al. Prediction on low carbon scenario by 2050 (in Chinese). Environ Prot, 2009, 24: 28-30

6 Chen W Y, Wu Z X, He J K. Two-convergence approach for future global carbon permit allocation (in Chinese). J Tsinghua (Sci \& Tech), 2005, 45: 850-853

7 Wang $\mathrm{Z}, \mathrm{Wu}$, $\mathrm{Li} \mathrm{G} \mathrm{Q}$, et al. Using simulation to assess climatechange strategies for global participation (in Chinese). Acta Ecol Sin, 2009, 29: 2407-2417

8 Nordhuas W D, Yang Z L. Regional dynamic general-equilibrium model of alternative climate-change strategies. Am Econ Rev, 1996, 86: 741-746

9 Margoluis R, Stern C. Using conceptual models as a planning and evaluation tool in conservation. Eval Prog Plann, 2009, 32: 138-147

10 Popp D. ENTICE: Endogenous backstop technology in the DICE model of global warming. J Environ Econ Manag, 2004, 48: 742-768

11 Wang Z, Jiang Y H, Wu J, et al. The research on China's potential abatement of $\mathrm{CO}_{2}$ by technological progress (in Chinese). Acta Ecol Sin, 2006, 26: 423-431

12 Stanton E A, Ackerman F, Kartha S. Inside the integrated assessment models: Four issues in climate economics. Clim Dev, 2009, 1: 166184

13 Wang Z, Zhu Q T, Wu J. Research on China's emission reduction scheme for searching superiority with uncertainty of climate change (in Chinese). Bull Chin Acad Sci, 2011, 5: 30-36

14 Douven R, Peeters M. GDP-spillovers in multi-country models. Econ Model, 1998, 15: 163-195

15 McKibbin W J, Sachs J D. Global Linkages: Macroeconomic Interdependence and Cooperation in the World Economy. Washington: Brookings Institution, 1991

16 Grossman G M, Helpman E. Trade, knowledge spillovers, and growth. Eur Econ Rev, 1991, 35: 517-526

17 Wang Z, Li H Q, Wu J, et al. Policy modeling on the GDP spillovers of carbon abatement polices between China and the United States. Econ Model, 2010, 20: 40-45

18 Barro R, Sala-i-Martin. Economic Growth. Cambridge: Massachusetts: MIT Press, 2004

19 Nordhaus W D. A review of the stern review on the economics of climate change. J Econ Lit, 2007, XLV: 703-724

20 Stern N. Key Elements of a Global Deal on Climate Change. London: London School of Economics and Political Science (LSE), 2008

Open Access This article is distributed under the terms of the Creative Commons Attribution License which permits any use, distribution, and reproduction in any medium, provided the original author(s) and source are credited.

\section{Supporting Information}

Table S1 Country classification

The supporting information is available online at csb.scichina.com and www.springerlink.com. The supporting materials are published as submitted, without typesetting or editing. The responsibility for scientific accuracy and content remains entirely with the authors. 